Preprint typeset in JHEP style. - PAPER VERSION

IU-MSTP/49

hep-lat/0206003

\title{
More about the axial anomaly on the lattice
}

\author{
Hiroshi Igarashi \\ Department of Mathematical Sciences, Ibaraki University, Mito 310-8512, Japan \\ E-mail: igarashi@serra.sci.ibaraki.ac.jp \\ Kiyoshi Okuyama \\ Department of Mathematical Sciences, Ibaraki University, Mito 310-8512, Japan \\ E-mail: okuyama@serra.sci.ibaraki.ac.jp \\ Hiroshi Suzuki \\ Department of Mathematical Sciences, Ibaraki University, Mito 310-8512, Japan \\ E-mail: hsuzuki@mito.ipc.ibaraki.ac.jp
}

\begin{abstract}
We study the axial anomaly defined on a finite-size lattice by using a Dirac operator which obeys the Ginsparg-Wilson relation. When the gauge group is $\mathrm{U}(1)$, we show that the basic structure of axial anomaly on the infinite lattice, which can be deduced by a cohomological analysis, persists even on (sufficiently large) finite-size lattices. For non-abelian gauge groups, we propose a conjecture on a possible form of axial anomaly on the infinite lattice, which holds to all orders in perturbation theory. With this conjecture, we show that a structure of the axial anomaly on finite-size lattices is again basically identical to that on the infinite lattice. Our analysis with the Ginsparg-Wilson Dirac operator indicates that, in appropriate frameworks, the basic structure of axial anomaly is quite robust and it persists even in a system with finite ultraviolet and infrared cutoffs.
\end{abstract}

KeYworDs: Renormalization, Regularization and Renormalons, Lattice Gauge Field Theories, Gauge Symmetry, Anomalies in Field and String Theories. 


\section{Introduction}

In ref. [1], Lüscher pointed out that a cohomological analysis can be used to determine a basic structure of the axial anomaly in abelian gauge theories with finite lattice spacings. This work paved a way to study the axial anomaly in a system with a finite ultraviolet cutoff and then the technique was applied for various cases $[2,3,4,5,6]$. The crucial properties which make this analysis possible are the locality, the gauge invariance and a topological property of the axial anomaly. The axial anomaly defined by the gauge covariant Dirac operator $[7,8]$ which satisfies the Ginsparg-Wilson relation [9], especially the overlap-Dirac operator [8], in fact possesses the required properties $[10,11,12,13,14]$. A further elaborate analysis with this recognition finally led to a non-perturbative construction of anomaly-free abelian chiral gauge theories on the lattice [15].

The cohomological analysis, however, is limited to the case of a lattice with an infinite size. A direct cohomological analysis for finite-size lattices is not feasible because (i) The analysis is based on the lattice Poincaré lemma [1], which is a lattice analogue of the Poincaré lemma being valid for $\mathbb{R}^{d}$. When the topology of the lattice is non-trivial (as is the case for the periodic lattice), one expects a non-trivial dcohomology on the lattice. (ii) The cohomology relevant to an analysis of axial anomaly is a local cohomology, for which the concept of the locality is vital. The meaning of the locality, however, is not clear on a lattice with a finite size because a Dirac operator which obeys the Ginsparg-Wilson relation has to have exponentially decaying tails $[16,17]$.

In this paper, we study the axial anomaly defined on a finite-size lattice by using the Ginsparg-Wilson Dirac operator. This analysis provides an approach to the axial anomaly in a system with ultraviolet and infrared cutoffs. As already noted, a direct generalization of the technique of ref. [1] is not feasible. Instead, we point out that it is possible to determine the structure of axial anomaly using an argument similar to that of ref. [15] at least in abelian gauge theories. For nonabelian theories, we propose a conjecture on a possible form of axial anomaly on the infinite lattice, which is correct within perturbation theory. Under this conjecture, a similar argument can be applied to non-abelian cases too. These results indicate that the structure of axial anomaly is quite robust even with ultraviolet and infrared cutoffs in appropriate formulations (in the present case, a formulation based on the Ginsparg-Wilson relation). We consider an even-dimensional lattice $\Gamma$ whose size is $L, \Gamma=\left\{x \in \mathbb{Z}^{d} \mid 0 \leq x_{\mu}<L\right\}$, and the gauge field $U(x, \mu) \in G$ (G is the gauge group) is assumed to be periodic on $\Gamma, U(x+L \hat{\nu}, \mu)=U(x, \mu) .{ }^{1}$ The lattice spacing $a$ is set to be unity, except when the classical continuum limit is considered.

\footnotetext{
${ }^{1} \hat{\mu}$ denotes the unit vector in direction $\mu$.
} 


\section{Preliminaries}

The axial anomaly for the Ginsparg-Wilson Dirac operator is defined by (see for example refs. [11, 12] for the background)

$$
\mathcal{A}(x)=\operatorname{tr} \gamma_{d+1}\left[1-\frac{1}{2} D(x, x)\right] .
$$

The kernel of the Dirac operator $D(x, y)$ satisfies the Ginsparg-Wilson relation

$$
\gamma_{d+1} D(x, y)+D(x, y) \gamma_{d+1}=D \gamma_{d+1} D(x, y)
$$

The salient feature of $\mathcal{A}(x)$ is a lattice analogue of the analytic index theorem [10]

$$
\sum_{x \in \Gamma} \mathcal{A}(x)=n_{+}-n_{-},
$$

which follows from the algebraic relation (2.2) alone; here $n_{+}\left(n_{-}\right)$is the number of zero-modes of $\gamma_{d+1} D$ with the positive (negative) chirality. The index theorem (2.3) implies that the Dirac operator cannot be a smooth function of the gauge field in general, because the configuration space of lattice gauge field is arcwise connected and, barring a possibility that $n_{+}-n_{-}$is constant for all configurations, the integer $n_{+}-n_{-}$jumps at certain points in the configuration space. A sufficient condition for the smoothness of the overlap-Dirac operator [8] is the admissibility $[13,14]$

$$
\|1-U(x, \mu, \nu)\|<\epsilon, \quad \text { for all } x, \mu, \nu,
$$

where $U(x, \mu, \nu)$ is the plaquette variable and $\epsilon$ is a constant smaller than (2 $\sqrt{2}) / d(d-1)\left[14 .^{2}\right.$ After imposing this admissibility, the space of allowed gauge field configurations may have non-trivial topology. This condition also guarantees the locality of the operator $[13,14]$

$$
\|D(x, y)\| \leq C\left(1+\|x-y\|^{p}\right) e^{-\|x-y\| / \varrho}
$$

where $C$ and $p$ are constants and $\varrho$ is a localization range of the Dirac operator. In addition to the gauge covariance and the locality of the Dirac operator, we assume that it has the same transformation law as the standard Wilson-Dirac operator under discrete symmetries of the lattice (rotations, reflections, etc.). In particular, we require the translational invariance, i.e., $D(x, y)$ is identical to $D(x+z, y+z)$ if the gauge field is shifted at the same time $U(x, \mu) \rightarrow U(x+z, \mu)$.

Suppose that we have constructed a Dirac operator on a lattice with the size $L$. When $L \rightarrow \infty, D(x, y)$ is promoted to a Dirac operator on the infinite lattice $D(x, y) \rightarrow D^{\infty}(x, y)$. This operator also obeys the Ginsparg-Wilson relation

$$
\gamma_{d+1} D^{\infty}(x, y)+D^{\infty}(x, y) \gamma_{d+1}=D^{\infty} \gamma_{d+1} D^{\infty}(x, y) .
$$

\footnotetext{
${ }^{2}$ When the mass parameter $m$ in the overlap-Dirac operator is unity, $|m|=1$.
} 
In what follows, when we compare objects on the finite lattice $\Gamma$ and on the infinite lattice, we always take repeated copies of a configuration of the gauge field on $\Gamma$ as the gauge-field configuration on the infinite lattice. $D(x, x)$ on $\Gamma$ and $D^{\infty}(x, x)$ with the argument $x$ restricted to $\Gamma$ may somewhat differ because they have exponentially decaying tails. However we assume that this "finite size correction" is exponentially small,

$$
\left\|D(x, x)-D^{\infty}(x, x)\right\| \leq \kappa L^{\nu} e^{-L / \varrho}, \quad \text { for } x \in \Gamma,
$$

where $\kappa$ and $\nu$ are constants. The overlap-Dirac operator [8] possesses all the required properties we assumed above. ${ }^{3}$

Now, on the infinite lattice, the axial anomaly is given by

$$
\mathcal{A}^{\infty}(x)=\operatorname{tr} \gamma_{d+1}\left[1-\frac{1}{2} D^{\infty}(x, x)\right] .
$$

This is a topological field in the sense that

$$
\sum_{x \in \mathbb{Z}^{d}} \delta \mathcal{A}^{\infty}(x)=0
$$

where $\delta$ denotes a local variation of the gauge field. This property can be shown from the Ginsparg-Wilson relation (2.6) (see ref. [2] for example). $\mathcal{A}^{\infty}(x)$ is thus a local topological gauge invariant pseudoscalar field. ${ }^{4}$ When the gauge group is $\mathrm{U}(1)$, we can then apply the cohomological analysis $[1,2]$ to this quantity. The result is ${ }^{5}$

$$
\mathcal{A}^{\infty}(x)=q(x)+\partial_{\mu}^{*} k_{\mu}^{\infty}(x),
$$

where $k_{\mu}^{\infty}(x)$ is a local gauge invariant axial vector current (which is translational invariant) and the topological density $q(x)$ is given by

$$
\begin{aligned}
q(x)=\frac{\mathcal{N} i^{d / 2}}{(4 \pi)^{d / 2}(d / 2) !} & \epsilon_{\mu_{1} \nu_{1} \cdots \mu_{d / 2} \nu_{d / 2}} F_{\mu_{1} \nu_{1}}(x) F_{\mu_{2} \nu_{2}}\left(x+\hat{\mu}_{1}+\hat{\nu}_{1}\right) \cdots \\
& \times F_{\mu_{d / 2} \nu_{d / 2}}\left(x+\hat{\mu}_{1}+\hat{\nu}_{1}+\cdots+\hat{\mu}_{d / 2-1}+\hat{\nu}_{d / 2-1}\right)
\end{aligned}
$$

with an integer $\mathcal{N}$. The abelian field strength is defined by ${ }^{6}$

$$
F_{\mu \nu}(x)=\ln U(x, \mu, \nu), \quad-\pi<\frac{1}{i} F_{\mu \nu}(x) \leq \pi .
$$

\footnotetext{
${ }^{3}$ For the overlap-Dirac operator, whose basic building block is the Wilson-Dirac operator, one can show the relation $D(x, y)=\sum_{n \in \mathbb{Z}^{d}} D^{\infty}(x, y+L n)$. We thank Yoshio Kikukawa and Martin Lüscher for clarifying this point. From this relation and the locality (2.5), one obtains the bound (2.7).

${ }^{4} \mathrm{~A}$ field $\phi(x)$ is termed local, when its dependence on the gauge field at a point $y$ is exponentially suppressed as $\|x-y\| \rightarrow \infty$. For a more precise definition, see ref. [1].

${ }^{5} \partial_{\mu}$ and $\partial_{\mu}^{*}$ denote the forward and the backward difference operators respectively:

$$
\partial_{\mu} f(x)=f(x+\hat{\mu})-f(x), \quad \partial_{\mu}^{*} f(x)=f(x)-f(x-\hat{\mu}) .
$$

${ }^{6}$ For the cohomological argument to apply, the constant $\epsilon$ in eq. (2.4) has to be smaller than 1 and $\left|F_{\mu \nu}(x) / i\right|<\pi / 3[1]$.
} 
Strictly speaking, the cohomological analysis alone admits a more general form of $q(x)$ than eq. (2.12); for example, $\beta_{\mu \nu} F_{\mu \nu}(x)$ with anti-symmetric constants $\beta_{\mu \nu}$ is also possible. However, since $\mathcal{A}^{\infty}(x)$ is a pseudoscalar under lattice rotations and reflections, one infers that it must be proportional to the Levi-Civita symbol. Also the numerical coefficient in eq. (2.12) is left undermined in the cohomological analysis. We can however use a matching with the result in the classical continuum limit; the integer $\mathcal{N}$ is given by a sum of chiral charges of massless degrees of freedom $[18,19,20,21,22,23,24]$.

Note that eq. (2.11) is a statement for finite lattice spacings. Eq. (2.11) states that, even when the lattice spacing is finite, the main part of the axial anomaly is given by the topological density $q(x)$ which has a quite analogous form to the continuum counterpart. On the other hand, the total divergence term $\partial_{\mu}^{*} k_{\mu}^{\infty}(x)$ represents "lattice artifacts" in the axial anomaly which depend on the details of the Dirac operator adopted. Our aim in this paper is to show or argue that the structure represented by eqs. (2.11) and (2.12) persists even on finite-size lattices and for general gauge groups $G$.

\section{Abelian gauge theory $G=\mathrm{U}(1)$}

For the axial anomaly defined on a finite lattice (2.1), a direct cohomological analysis is not feasible. Nevertheless, we can show the following

Theorem 3.1 When $G=\mathrm{U}(1)$, if the lattice is sufficiently large compared to the localization range @ of the Dirac operator, say $L / \varrho \geq n$,

$$
\mathcal{A}(x)=q(x)+\partial_{\mu}^{*} k_{\mu}(x)
$$

where $k_{\mu}(x)$ is a gauge invariant periodic current on $\Gamma$. The current $k_{\mu}(x)$ moreover satisfies the bound

$$
\left|k_{\mu}(x)-k_{\mu}^{\infty}(x)\right| \leq \kappa_{1} L^{\nu_{1}} e^{-L / \varrho}
$$

with constants $\kappa_{1}$ and $\nu_{1}$.

We emphasize that, for a sufficiently large $L$, eq. (3.1) is an exact statement for the axial anomaly $\mathcal{A}(x)$. Eq. (3.2) shows that the current $k_{\mu}(x)$ differs from the local current $k_{\mu}^{\infty}(x)$ defined on the infinite lattice only by an exponentially small amount. Hence, when the lattice size becomes large compared to $\varrho$ and thus when the concept of the locality becomes meaningful, the current $k_{\mu}(x)$ can be regarded as a local current. In this way, eq. (3.1) shows that the structure of axial anomaly on finite-size lattices is basically identical to that on the infinite lattice (2.11). The validity of this theorem has been argued intuitively by Chiu [25]. 
PROOF. The configuration space of the gauge fields allowed by the admissibility (2.4) consists of many components. Each component is uniquely characterized [15] by the magnetic flux

$$
m_{\mu \nu}=\frac{1}{2 \pi i} \sum_{s, t=0}^{L-1} F_{\mu \nu}(x+s \hat{\mu}+t \hat{\nu}),
$$

which is an integer. For a configuration with the magnetic flux $m_{\mu \nu}$, from eq. (2.12), one has $[26]$

$$
\begin{aligned}
\sum_{x \in \Gamma} \mathcal{A}^{\infty}(x) & =\sum_{x \in \Gamma} q(x)=\frac{\mathcal{N}(-1)^{d / 2}}{2^{d / 2}(d / 2) !} \epsilon_{\mu_{1} \nu_{1} \cdots \mu_{d / 2} \nu_{d / 2}} m_{\mu_{1} \nu_{1}} m_{\mu_{2} \nu_{2}} \cdots m_{\mu_{d / 2} \nu_{d / 2}} \\
& =\text { an integer, }
\end{aligned}
$$

where the first equality follows from the translational invariance of $k_{\mu}^{\infty}(x)$ (namely, $k_{\mu}^{\infty}(x)$ is a periodic current on $\Gamma$, when the gauge field is periodic). ${ }^{7}$ Combined with the index theorem (2.3), we see that $\sum_{x \in \Gamma} \mathcal{A}(x)-\sum_{x \in \Gamma} \mathcal{A}^{\infty}(x)$ is an integer. This integer is however bounded by an exponentially small quantity: From the assumed property (2.7), one infers that

$$
\sum_{x \in \Gamma}\left[\mathcal{A}(x)-\mathcal{A}^{\infty}(x)\right] \leq \kappa_{2} L^{\nu_{2}} e^{-L / \varrho}
$$

Therefore, when $L$ is greater than some multiple of $\varrho$, one has

$$
\sum_{x \in \Gamma}\left[\mathcal{A}(x)-\mathcal{A}^{\infty}(x)\right]=0
$$

For this, we can apply the following

Lemma 3.1 For a periodic field $c(x)$ on $\Gamma$ satisfying

$$
\sum_{x \in \Gamma} c(x)=0
$$

there exists a periodic current $b_{\mu}(x)$ which is given by a sum of $c(y)$, the precise meaning of which is given in eq. (3.9) below, such that

$$
\partial_{\mu}^{*} b_{\mu}(x)=c(x), \quad\left|b_{\mu}(x)\right| \leq 2 L \max _{x \in \Gamma}|c(x)| .
$$

Applying this lemma to eq. (3.6), we see that there exists a gauge invariant periodic current $\Delta k_{\mu}(x)$ such that $\mathcal{A}(x)-\mathcal{A}^{\infty}(x)=\partial_{\mu}^{*} \Delta k_{\mu}(x)$. This field is exponentially small, $\left|\Delta k_{\mu}(x)\right| \leq \kappa_{1} L^{\nu_{1}} e^{-L / \varrho}$, thus $k_{\mu}(x)=k_{\mu}^{\infty}(x)+\Delta k_{\mu}(x)$ which proves the theorem.

\footnotetext{
${ }^{7}$ Eq. (2.3) and theorem (3.1) show that the index is given by the combination (3.4) in terms of the magnetic flux. For the overlap-Dirac operator, this relation has been verified numerically for $d=2$ and $d=4[27,28]$ and proven analytically for $d=2[28]$.
} 
The assertions of the lemma immediately follow from the explicit construction of $b_{\mu}(x)$ (though this is not unique):

$$
\begin{aligned}
b_{\mu}(x)=\frac{1}{L^{d-\mu}} \sum_{y_{\mu}=0}^{x_{\mu}} & \sum_{y_{\mu+1}=0}^{L-1} \cdots \sum_{y_{d}=0}^{L-1} c\left(x_{1}, \ldots, x_{\mu-1}, y_{\mu}, \ldots, y_{d}\right) \\
& -\frac{x_{\mu}+1}{L^{d-\mu+1}} \sum_{y_{\mu}=0}^{L-1} \cdots \sum_{y_{d}=0}^{L-1} c\left(x_{1}, \ldots, x_{\mu-1}, y_{\mu}, \ldots, y_{d}\right) .
\end{aligned}
$$

Note that since $b_{\mu}(x)$ is given by a sum of the field $c(x), b_{\mu}(x)$ is gauge invariant if so is $c(x)$.

\section{Non-abelian cases}

For general gauge groups $G$, a cohomological argument in a non-perturbative level is not known even on the infinite lattice. Thus we propose a conjecture on a possible form of $\mathcal{A}^{\infty}(x)$ :

Conjecture 4.1 For general $G$,

$$
\mathcal{A}^{\infty}(x)=q(x)+\partial_{\mu}^{*} k_{\mu}^{\infty}(x)
$$

where $k_{\mu}^{\infty}(x)$ is a local gauge invariant axial vector current (which is translational invariant) and the topological density $q(x)$ is given by Lüscher's topological density [29] and its higher dimensional extensions.

The explicit expression of Lüscher's topological density is known only for $d=2$ and for $d=4$ : In our context, it is given by $\mathcal{N}$ times eq. (32) of ref. [29]. We simply assume that the construction can be pursued for higher dimensional cases. ${ }^{8}$ The construction of ref. [29] does not provide a pseudoscalar $q(x)$. However, we may always enforce this pseudoscalar property by taking average over lattice symmetries; we assume that this has been done and $q(x)$ is a pseudoscalar. The topological density has the classical continuum limit

$$
\lim _{a \rightarrow 0} \frac{1}{a^{d}} q(x)=\frac{\mathcal{N} i^{d / 2}}{(4 \pi)^{d / 2}(d / 2) !} \epsilon_{\mu_{1} \nu_{1} \cdots \mu_{d / 2} \nu_{d / 2}} \operatorname{tr} F_{\mu_{1} \nu_{1}} F_{\mu_{2} \nu_{2}} \cdots F_{\mu_{d / 2} \nu_{d / 2}}(x) .
$$

At the moment, we cannot prove the above conjecture in the non-perturbative level. However, we see that the conjecture holds to all orders in perturbation theory; the following theorem guarantees that a gauge invariant topological field is unique (up to a total divergence) under certain conditions:

\footnotetext{
${ }^{8}$ For $G=\mathrm{U}(1)$, the construction of ref. [29] can be generalized to arbitrary dimensions [26]. The equivalence of eq. (4.1) with eq. (2.11) for $G=\mathrm{U}(1)$ has been shown [26]. See also ref. [30].
} 
Theorem 4.1 Let $p(x)$ be a local gauge invariant pseudoscalar field (which is translational invariant) on the infinite lattice whose dependences on the lattice spacing a arise only though the gauge field. ${ }^{9}$ If it is topological

$$
\sum_{x \in \mathbb{R}^{d}} \delta p(x)=0
$$

and the classical continuum limit $\lim _{a \rightarrow 0} p(x) / a^{d}$ vanishes, then to all orders in perturbation theory,

$$
p(x)=\partial_{\mu}^{*} \ell_{\mu}(x)
$$

where $\ell_{\mu}(x)$ is a local gauge invariant axial vector current.

Proof. Our proof is rather similar to the cohomological argument of ref. [5]. We expand $p(x)$ with respect to the bare gauge coupling constant $g_{0}$ introduced by $U(x, \mu)=e^{g_{0} A_{\mu}(x)}$

$$
\begin{aligned}
& p(x)=\sum_{k=1}^{\infty} p^{(k)}(x), \\
& p^{(k)}(x)=\frac{g_{0}^{k}}{k !} \sum_{y_{1}, \ldots, y_{k}} p^{(k)}\left(x, y_{1}, \ldots, y_{k}\right)_{\mu_{1} \cdots \mu_{k}}^{a_{1} \cdots a_{k}} A_{\mu_{1}}^{a_{1}}\left(y_{1}\right) \cdots A_{\mu_{k}}^{a_{k}}\left(y_{k}\right),
\end{aligned}
$$

where $A_{\mu}(x)=A_{\mu}^{a}(x) T^{a}$.

First consider $p^{(1)}(x)$. Since $p(x)$ is gauge invariant, $p^{(1)}(x)$ is invariant under the linearized gauge transformation

$$
A_{\mu}(x) \rightarrow A_{\mu}(x)+\partial_{\mu} \omega(x),
$$

and also under the constant gauge transformation

$$
A_{\mu}(x) \rightarrow A_{\mu}(x)+\left[\omega, A_{\mu}(x)\right]
$$

Moreover, since $p^{(1)}(x)$ is a local topological pseudoscalar field and eq. (4.6) is the gauge transformation in abelian theory, one can invoke the cohomological analysis in abelian theory. The result is

$$
p^{(1)}(x)=\partial_{\mu}^{*} \ell_{\mu}^{(1)}(x), \quad \ell_{\mu}^{(1)}(x)=g_{0} \sum_{y} \ell_{\mu}^{(1)}(x, y)_{\nu}^{a} A_{\nu}^{a}(y)
$$

The local axial vector current $\ell_{\mu}^{(1)}(x)$ is invariant under eqs. (4.6) and (4.7). A key observation is that, from $\ell_{\mu}^{(1)}(x)$, one can construct a field $\hat{\ell}_{\mu}^{(1)}(x)$ such that it is invariant under the original non-abelian gauge transformation and its lowest-order

\footnotetext{
${ }^{9}$ Recall that in the classical continuum limit the gauge potential is introduced as $U(x, \mu)=$ $\mathcal{P} \exp \left[a \int_{0}^{1} \mathrm{~d} t A_{\mu}(x+(1-t) a \hat{\mu})\right]$ where $a$ is the lattice spacing.
} 
$O\left(g_{0}\right)$ term coincides with $\ell_{\mu}^{(1)}(x)$. This can be accomplished by substituting the gauge potential $A_{\mu}^{a}(y)$ in eq. (4.8) by the expression [5]

$$
\widehat{A}_{\mu}^{a}(x, y)=\frac{2}{g_{0}} \operatorname{tr}\left\{T^{a}\left[1-W(x, y) U(y, \mu) W(x, y+\hat{\mu})^{-1}\right]\right\}
$$

where $W(x, y)$ is the ordered product of the link variables from $y$ to $x$ along the shortest path that goes first in direction 1, then direction 2, and so on. Note that $\widehat{A}_{\mu}^{a}(x, y)$ behaves gauge covariantly under the original non-abelian gauge transformation. Thus the resulting expression,

$$
\widehat{\ell}_{\mu}^{(1)}(x)=g_{0} \sum_{y} \ell_{\mu}^{(1)}(x, y)_{\nu}^{a} \widehat{A}_{\nu}^{a}(x, y)
$$

is invariant under the non-abelian gauge transformation due to the invariance of $\ell_{\mu}^{(1)}(x)$ under eq. (4.7). Moreover, since

$$
\widehat{A}_{\mu}(x, y)=A_{\mu}(y)+\partial_{\mu}^{y} \omega(x, y)+O\left(g_{0}\right)
$$

with $\omega(x, y)$ the oriented line sum of the gauge potential from $y$ to $x$, the invariance under eq. (4.6) implies that $\widehat{\ell}_{\mu}^{(1)}(x)=\ell_{\mu}^{(1)}(x)+O\left(g_{0}^{2}\right) \cdot{ }^{10}$ Using $\widehat{\ell}_{\mu}^{(1)}(x)$, we may define a local gauge invariant pseudoscalar field

$$
p(x)-\partial_{\mu}^{*} \widehat{\ell}_{\mu}^{(1)}(x) .
$$

which has identical properties with $p(x)$ except that it starts with $O\left(g_{0}^{2}\right)$ term. Thus we can repeat the above argument from eq. (4.5) for the field (4.12). This time, however, the perturbation series analogous to eq. (4.5) starts from $k=2$.

In this way, we repeat the steps from eq. (4.5) to eq. (4.12) by eliminating the lowest-order term of the topological field until the first order term becomes $O\left(g_{0}^{d / 2}\right)$; here a new situation arises. The cohomological analysis (with the fact that it is a pseudoscalar) tells that

$$
\begin{array}{ll}
c^{a_{1} \cdots a_{d / 2}} \epsilon_{\mu_{1} \nu_{1} \cdots \mu_{d / 2} \nu_{d / 2}} & F_{\mu_{1} \nu_{1}}^{a_{1}}(x) F_{\mu_{2} \nu_{2}}^{a_{2}}\left(x+\hat{\mu}_{1}+\hat{\nu}_{1}\right) \cdots \\
& \times F_{\mu_{d / 2} \nu_{d / 2}}^{a_{d / 2}}\left(x+\hat{\mu}_{1}+\hat{\nu}_{1}+\cdots+\hat{\mu}_{d / 2-1}+\hat{\nu}_{d / 2-1}\right) \\
& \\
+\partial_{\mu}^{*} \ell_{\mu}^{(d / 2)}, &
\end{array}
$$

$\left[F_{\mu \nu}^{a}(x)=\partial_{\mu} A_{\nu}^{a}(x)-\partial_{\nu} A_{\mu}^{a}(x)\right.$ denotes the linearized field strength $]$ is a possible form of $p(x)-\sum_{k=1}^{d / 2-1} \partial_{\mu}^{*} \widehat{\ell}_{\mu}^{(k)}(x)$. However, since the continuum limit of $p(x), \lim _{a \rightarrow 0} p(x) / a^{d}$ vanishes, we infer that the constants $c^{a_{1} \cdots a_{d / 2}}$ vanish, $c^{a_{1} \cdots a_{d / 2}}=0$. Thus we again have a total divergence. Further repeating the above procedure, we finally establish $p(x)=\partial_{\mu}^{*} \sum_{k=1}^{\infty} \widehat{\ell}_{\mu}^{(k)}(x)$.

\footnotetext{
${ }^{10}$ The current $\widehat{\ell}_{\mu}^{(1)}(x)$ so constructed is not an axial vector under the lattice symmetries. However we can always enforce this by taking average over lattice symmetries.
} 
Going back to eq. (4.1), we note that both $\mathcal{A}^{\infty}(x)$ and $q(x)$ are a local gauge invariant topological pseudoscalar field (for the latter, those properties follow from the construction of $q(x)[29])$. Moreover, they have the same classical continuum limit (4.2). Thus, applying theorem 4.1 to $\mathcal{A}^{\infty}(x)-q(x)$, we see that the conjecture holds to all orders in perturbation theory.

Now, in the proof of theorem 3.1 in abelian theory, every steps are valid even for non-abelian theories, except for the crucial relation (3.4), namely $\sum_{x \in \Gamma} \mathcal{A}^{\infty}(x)$ is an integer. With our conjecture 4.1 for non-abelian cases, this last condition is also satisfied; $\sum_{x \in \Gamma} q(x)$ is Lüscher's topological charge on a periodic lattice which is an integer. So, repeating the proof for theorem 3.1, we have

Corollary of conjecture 4.1 For general $G$, if the lattice is sufficiently large compared to the localization range @ of the Dirac operator, say $L / \varrho \geq n$,

$$
\mathcal{A}(x)=q(x)+\partial_{\mu}^{*} k_{\mu}(x)
$$

where $k_{\mu}(x)$ is a gauge invariant periodic current on $\Gamma$. The current $k_{\mu}(x)$ moreover satisfies the bound

$$
\left|k_{\mu}(x)-k_{\mu}^{\infty}(x)\right| \leq \kappa_{1} L^{\nu_{1}} e^{-L / \varrho},
$$

with constants $\kappa_{1}$ and $\nu_{1}$. The topological density $q(x)$ is given by Lüscher's topological density [29] and its higher dimensional extensions.

This corollary states that the basic structure of axial anomaly on finite lattices is identical that on the infinite lattice. Summing eq. (4.14) over the lattice $\Gamma$, one has an equality between the index of the Dirac operator (2.3) and the geometricallydefined lattice topological charge [29]. This equivalence ("lattice index theorem") has been thought to be true for long time since the analyses in refs. [27, 31]. Our argument provides a further support for this equivalence.

\section{Conclusion}

In this paper, we have studied the axial anomaly defined on a finite-size lattice by using a Ginsparg-Wilson Dirac operator. For $G=\mathrm{U}(1)$, we show that the basic structure of axial anomaly on the infinite lattice, which has a quite analogous form to the continuum counterpart, persists even on a sufficiently large finite-size lattices. For general $G$, we conjectured that the axial anomaly on the infinite lattice is basically given by Lüscher's topological density; actually this holds to all orders in perturbation theory. With this conjecture, we showed that this structure again persists even on finite-size lattices. Since Lüscher's topological density is a geometrically natural definition of the Chern form in lattice gauge theory (note that it is proportional to $\operatorname{str} T^{a_{1}} \cdots T^{a_{d / 2}}$ ), our analysis indicates that the basic structure of axial anomaly in continuum theory is quite robust and it persists even in a system with finite 
ultraviolet and infrared cutoffs. Of course, we indicated this persistency only in a framework with the Ginsparg-Wilson relation. To understand precise conditions on the formulation for this persistency to hold is an interesting open question; for example, one may enlarge the set of formulations by using the generalized GinspargWilson relation [32].

In the gauge invariant lattice formulation of abelian chiral gauge theories [15], a knowledge on the structure of $\mathrm{U}(1)$ gauge anomaly on finite-size lattices was of crucial importance. Recalling this fact, we believe that our analyses will be useful in extending the construction of ref. [15] to non-abelian gauge theories.

H.S. would like to thank Takanori Fujiwara, Takahiro Fukui, Yoshio Kikukawa and Martin Lüscher for valuable discussions. We are grateful to Kazuo Fujikawa for a careful reading of the manuscript.

\section{References}

[1] M. Lüscher, Topology and the axial anomaly in abelian lattice gauge theories, Nucl. Phys. B 538 (1999) 515 [hep-lat/9808021].

[2] T. Fujiwara, H. Suzuki and K. Wu, Noncommutative differential calculus and the axial anomaly in abelian lattice gauge theories, Nucl. Phys. B 569 (2000) 643 [hep-lat/9906015]; Axial anomaly in lattice abelian gauge theory in arbitrary dimensions, Phys. Lett. B 463 (1999) 63 [hep-lat/9906016].

[3] H. Suzuki, Anomaly cancellation condition in lattice gauge theory, Nucl. Phys. B 585 (2000) 471 [hep-lat/0002009];

H. Igarashi, K. Okuyama and H. Suzuki, Errata and addenda to "Anomaly cancellation condition in lattice gauge theory", hep-lat/0012018.

[4] Y. Kikukawa and Y. Nakayama, Gauge anomaly cancellation in $\mathrm{SU}(2)_{L} \times \mathrm{U}(1)_{Y}$ electroweak theory on the lattice, Nucl. Phys. B 597 (2001) 519 [hep-lat/0005015].

[5] M. Lüscher, Lattice regularization of chiral gauge theories to all orders of perturbation theory, J. High Energy Phys. 06 (2000) 028 [hep-lat/0006014].

[6] Y. Kikukawa, Domain wall fermion and chiral gauge theories on the lattice with exact gauge invariance, Phys. Rev. D 65 (2002) 074504 [hep-lat/0105032].

[7] P. Hasenfratz, Prospects for perfect actions, Nucl. Phys. 63 (Proc. Suppl.) (1998) 53 [hep-lat/9709110]; Lattice QCD without tuning, mixing and current renormalization, Nucl. Phys. B 525 (1998) 401 [hep-lat/9802007].

[8] H. Neuberger, Exactly massless quarks on the lattice, Phys. Lett. B 417 (1998) 141 [hep-lat/9707022]; More about exactly massless quarks on the lattice, Phys. Lett. B 427 (1998) 353 [hep-lat/9801031]. 
[9] P. H. Ginsparg and K. G. Wilson, A remnant of chiral symmetry on the lattice, Phys. Rev. D 25 (1982) 2649.

[10] P. Hasenfratz, V. Laliena and F. Niedermayer, The index theorem in QCD with a finite cut-off, Phys. Lett. B 427 (1998) 125 [hep-lat/9801021].

[11] M. Lüscher, Exact chiral symmetry on the lattice and the Ginsparg-Wilson relation, Phys. Lett. B 428 (1998) 342 [hep-lat/9802011].

[12] F. Niedermayer, Exact chiral symmetry, topological charge and related topics, Nucl. Phys. 73 (Proc. Suppl.) (1999) 105 [hep-lat/9810026].

[13] P. Hernández, K. Jansen and M. Lüscher, Locality properties of Neuberger's lattice Dirac operator, Nucl. Phys. B 552 (1999) 363 [hep-lat/9808010].

[14] H. Neuberger, Bounds on the Wilson Dirac operator, Phys. Rev. D 61 (2000) 085015 [hep-lat/9911004].

[15] M. Lüscher, Abelian chiral gauge theories on the lattice with exact gauge invariance, Nucl. Phys. B 549 (1999) 295 [hep-lat/9811032].

[16] I. Horvath, Ginsparg-Wilson relation and ultralocality, Phys. Rev. Lett. 81 (1998) 4063 [hep-lat/9808002]; Ginsparg-Wilson-Lüscher symmetry and ultralocality, Phys. Rev. D 60 (1999) 034510 [hep-lat/9901014].

[17] W. Bietenholz, On the absence of ultralocal Ginsparg-Wilson fermions, hep-lat/9901005.

[18] Y. Kikukawa and A. Yamada, Weak coupling expansion of massless QCD with a Ginsparg-Wilson fermion and axial U(1) anomaly, Phys. Lett. B 448 (1999) 265 [hep-lat/9806013].

[19] K. Fujikawa, A continuum limit of the chiral Jacobian in lattice gauge theory, Nucl. Phys. B 546 (1999) 480 [hep-th/9811235].

[20] D. H. Adams, Axial anomaly and topological charge in lattice gauge theory with overlap-Dirac operator, Ann. Phys. (NY) 296 (2002) 131 [hep-lat/9812003]; On the continuum limit of fermionic topological charge in lattice gauge theory, J. Math. Phys. 42 (2001) 5522 [hep-lat/0009026].

[21] H. Suzuki, Simple evaluation of chiral Jacobian with the overlap Dirac operator, Prog. Theor. Phys. 102 (1999) 141 [hep-th/9812019].

[22] T. W. Chiu and T. H. Hsieh, Perturbation calculation of the axial anomaly of Ginsparg-Wilson fermion, hep-lat/9901011.

[23] T. Reisz and H. J. Rothe, The axial anomaly in lattice QED: A universal point of view, Phys. Lett. B 455 (1999) 246 [hep-lat/9903003]. 
[24] M. Frewer and H. J. Rothe, Universality of the axial anomaly in lattice QCD, Phys. Rev. D 63 (2001) 054506 [hep-lat/0004005].

[25] T. W. Chiu, The axial anomaly of Ginsparg-Wilson fermion, Phys. Lett. B 445 (1999) 371 [hep-lat/9809013].

[26] T. Fujiwara, H. Suzuki and K. Wu, Topological charge of lattice Abelian gauge theory, Prog. Theor. Phys. 105 (2001) 789 [hep-lat/0001029].

[27] R. Narayanan and H. Neuberger, Chiral fermions on the lattice, Phys. Rev. Lett. 71 (1993) 3251 [hep-lat/9308011]; A construction of lattice chiral gauge theories, Nucl. Phys. B 443 (1995) 305 [hep-th/9411108].

[28] T. Fujiwara, A numerical study of spectral flows of Hermitian Wilson-Dirac operator and the index theorem in Abelian gauge theories on finite lattices, Prog. Theor. Phys. 107 (2002) 163 [hep-lat/0012007];

H. Kurokawa and T. Fujiwara, Spectrum of the hermitian Wilson-Dirac operator for a uniform magnetic field in two dimensions, hep-lat/0206014.

[29] M. Lüscher, Topology of lattice gauge fields, Comm. Math. Phys. 85 (1982) 39.

[30] A. Phillips, Characteristic numbers of U(1) valued lattice gauge fields, Ann. Phys. (NY) 161 (1985) 399.

[31] R. Narayanan and P. Vranas, A numerical test of the continuum index theorem on the lattice, Nucl. Phys. B 506 (1997) 373 [hep-lat/9702005].

[32] K. Fujikawa, Algebraic generalization of the Ginsparg-Wilson relation, Nucl. Phys. B 589 (2000) 487 [hep-lat/0004012];

K. Fujikawa and M. Ishibashi, Chiral anomaly for a new class of lattice Dirac operators, Nucl. Phys. B 587 (2000) 419 [hep-lat/0005003]; Locality properties of a new class of lattice Dirac operators, Nucl. Phys. B 605 (2001) 365 [hep-lat/0102012]; A perturbative study of a general class of lattice Dirac operators, Phys. Rev. D 65 (2002) 114504 [hep-lat/0201016]. 